\title{
Application of Deep Learning in Medical Image Processing - A Comprehensive Review
}

\author{
Aruna.G \\ Lecturer, Department of Software Engineering, Kiriom Institute of Technology, Cambodia ${ }^{1}$
}

\begin{abstract}
Deep learning could be a machine learning technique that teaches computers to try to to what comes naturally to humans. the essential concepts and models in deep learning have consequent from the synthetic neural network, which mimic human brain's activity pattern to intelligent the algorithms and save deadly human labour. Whether or not deep learning is an promising subfield newly from machine learning, it's enormous utilizations distribution from machine vision, voice and signal processing, sequence and text prediction, and Image processing topics, altogether shaping the productive Artificial Intelligence (AI) fields. This assessment introduces machine learning algorithms as applied to medical image analysis, that specialize in convolutional neural networks, and emphasizing clinical aspects of the sector. The good thing about machine learning in an exceedingly period of medical big data is that considerable hierarchal dealings within the information may be discovered algorithmically exclusive of laborious hand-crafting of features. The various significant challenges have emerged; along with the way to analyze massive datasets and the way to mine and extract new information on image Processing. This paper review the status of the Deep learning and their applications in Image processing field.
\end{abstract}

Keywords: Deep learning, Machine learning, Artificial Intelligence and Image processing.

\section{INTRODUCTION}

Deep learning may be a machine learning performance that teaches computers to try to to what comes naturally to humans: learn by example. Deep learning could be a key technology at the rear driverless cars, enabling them to be accustomed to a stop sign, or to differentiate a pedestrian from a lamppost. it's the key to voice control in consumer devices like phones, tablets, TVs, and hands-free speakers. Deep learning is getting many awareness lately and permanently reason. It's achieving results that weren't possible before. In deep learning, a computer model learns to perform classification tasks directly from images, text, or sound. Deep learning models can do state-of-the-art accuracy, sometimes beyond human-level performance. Models are trained by employing a large set of labeled data and neural net work architectures that enclose many layers. The earliest computing was firstly implemented on hardware system within the 1950s. The newer perception with the more systematic theorems, named machine learning, appeared within the 1960s. And its newly evolved division, deep learning, was first spoken round the 2000s, and shortly led to speedy applications in dissimilar fields, because of its extraordinary prediction performance on big data (Le Cun et al .,2015).

The basic concepts and models in deep learning have derived from the bogus neural network, which mimic human brain's activity pattern to intelligentize the algorithms and save tedious human labor (Mamoshina et al .,2016 and Mnih et al., 2015). While deep learning is an emerging subfield newly from machine learning, it's immense utilizations spreading from machine vision, voice and signal processing, sequence and text prediction, and computational biology topics, altogether shaping the useful AI fields (Esteva et al .,2017; Zhang et al.,2016 and Ching et al .,2018).

Medical imaging is that the broad-spectrum name given to the group of techniques and processes developed for creating anatomical or functional images of soma (partially or as a whole), which are used for both clinical and scientific purposes. Medical image analysis is one among the foremost significant studies within the field of medication, since results gained by the analysis lead field professionals for diagnosis, treatment planning, and verification of administered treatment. Moreover, recent developments in medical imaging methods and medical image processing techniques provided a major reduction within the requirement for invasive intervention in treatment of a spread of diseases or abnormalities.

In medical imaging the interest in deep learning is generally triggered by Convolutional Neural Networks (CNNs), a strong thanks to learn useful representations of images and other structured data. Before it became possible to use CNNs efficiently, these features typically had to be engineered by hand, or created by less powerful machine learning models. Once it became possible to use features learned directly from the info, many of the handcrafted image features were typically left by the wayside as they clad to be almost worthless compared to feature detectors found by CNNs. 
Vol. 9, Issue 6, June 2020

There are some strong preferences embedded in CNNs based Diagnostic radiography nominates the methodological conditions of medical imaging and it appropriates the training of medical images.

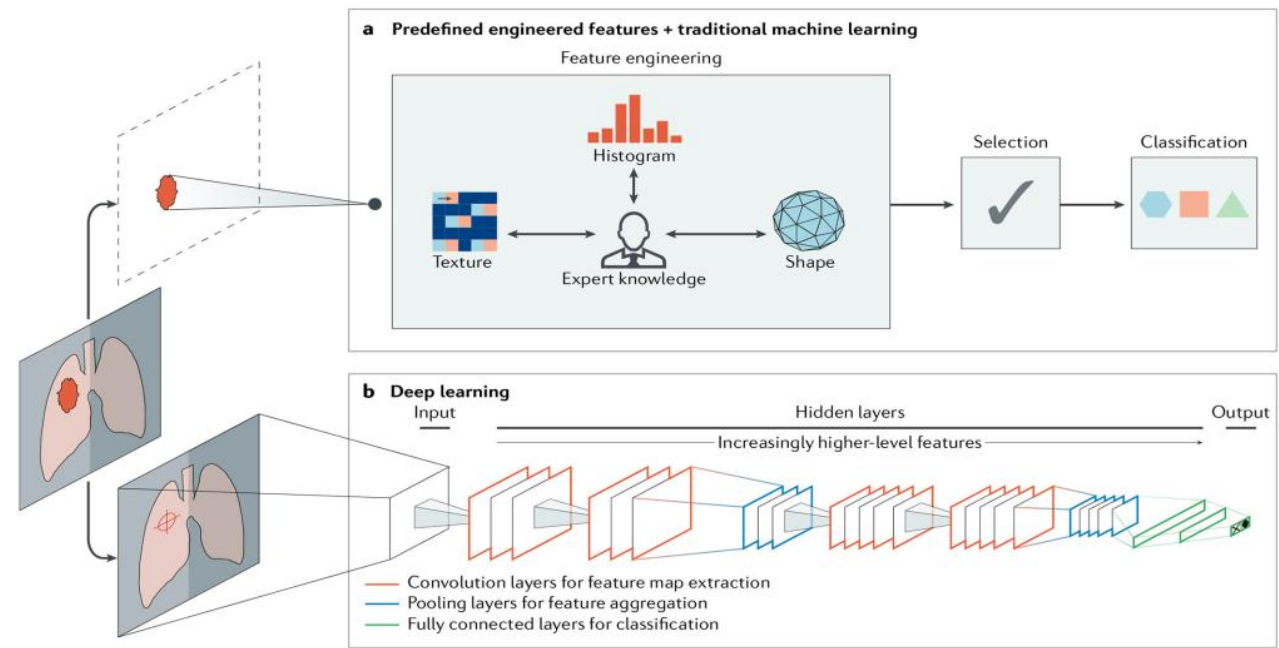

Figure 1. Machine learning model for medical image.

The radiographer is generally accountable for acquiring medical images of diagnostic aspect, although some radiological intervention is performed by radiologists (LeCun et al.,1998).

In the field of observation finding, medical imaging comprises a sub-control of biomedical engineering, medical physics or medicine betting on the context research and development within the area of two instrumentation, image acquisition, modelling and accounting are usually the bottle of biomedical engineering, medical physics and engineering science. Research into the function and perception of medical images are usually to preserve the radiology and therefore the medical sub-discipline relevant to the realm of bioscience under investigation. Many of the techniques matured for medical imaging even have scientific and industrial applications.

\section{DEEP LEARNING ALGORITHMS}

Recent advances in AI research have given rise to new, non-deterministic, deep learning algorithms that don't require explicit feature definition, representing a fundamentally different paradigm in machine learning (Le Cun et al 2015 and Kevin Zhou et al.,2017). The underlying methods of deep learning have existed for many years. However, only in recent years have sufficient data and computational power become available. Without explicit feature predefinition or selection, these algorithms learn directly by navigating the info space, giving them superior problem-solving capabilities. While various deep learning architectures are explored to handle different tasks, convolutional neural networks (CNNs) are the foremost prevalent deep learning architecture typologies in medical imaging today (Litjens,2017). A typical CNN comprises a series of layers that successively map image inputs to desired end points while learning increasingly higher-level imaging features. ranging from an input image, 'hidden layers' within CNNs usually include a series of convolution and pooling operations extracting feature maps and performing feature aggregation, respectively. These hidden layers are then followed by fully connected layers providing high-level reasoning before an output layer produces predictions. CNNs are often trained end-to-end with labelled data for supervised learning. Other architectures, like deep autoencoders 96 and generative adversarial networks 95, are more fitted to unsupervised learning tasks on unlabelled data. Transfer learning, or using pre-trained networks on other data sets, is usually utilized when addressing scarce data. (Shin,2016)

\section{TYPES OF MACHINE LEARNING}

Machine learning is majorly categorized into three types: supervised learning, unsupervised learning, and reinforcement learning

A. Supervised Learning: The supervised machine learning algorithms are those algorithms which needs external assistance. The input dataset is split into train and test dataset. The train dataset has output variable which must be predicted or classified. All algorithms learn some quite patterns from the training dataset and apply them to the test dataset for prediction or classification (Kotsiantis, 2007) 
B. Unsupervised Learning: The unsupervised learning algorithms learns few features from the information. When new data is introduced, it uses the previously learned features to acknowledge the category of the info. it's mainly used for clustering and have reduction.

C. Reinforcement Learning: Reinforcement learning could be a style of learning which makes decisions supported which actions to require such the result is more positive. The learner has no knowledge which actions to require until it's been given a situation. The action which is taken by the learner may affect situations and their actions within the future. Reinforcement learning solely depends on two criteria: trial and error search \& delayed outcome (Sutton, 1992).

\section{MACHINE LEARNING TOOLS}

Cell Profiler: Few years ago, software for biological image analysis only measured single parameter from group of images. As, in 2005, a computational biologist, Anne Carpenter from MIT and Harvard released a software called Cell Profiler for the measurement of quantitatively individual features like fuorescent cell number in microscopy field. But, currently Cell Profiler can produce thousands of features by implementing deep learning techniques.

Deep Variant: Application of deep learning is extensively employed in tools for mining genome data. Verily natural science and Google developed a tool supported deep learning called DeepVariant that predicts a standard kind of genetic variation more accurately as compared to traditional tools.

Atomwise: Another field is drug discovery during which deep learning contributing significantly. A San Francisco based biotech company called Atom wise has developed a algorithm that help to convert molecules into 3D pixels. This representation helps to account the 3D structure of proteins and tiny molecules with atomic precision. Then by using these features algorithm can predict small molecules that possibly interact with given protein (Webb ,2018).

Different types of deep learning methods exist like deep neural network (DNN), recurrent neural network (RNN), convolution neural network (CNN), deep autoencoder (DA), deep Boltzman machine (DBM), deep belief network $(\mathrm{DBN})$ and deep residual network (DRN) etc. within the field of biology some methods like, DNN, RNN, CNN, DA and DBM are most typically used methods (Mahmud et al.,2018). Translation of biological data to perform validation of biomarkers that reveal disease state could be a key task in biomedicine. DNN plays significant role within the identification of potential biomarkers from genome and proteome data. Deep learning also play important role in drug discovery (Mamoshina et al .,2016).

CNN has been used recently developed computational tool DeepCpG to predict DNA methylation states in single cells. within the DNA methylation, methyl groups related to DNA molecule and alter the functions of DNA molecule with causing any changes in sequence. DeepCpG also used for the prediction of known motifs that are chargeable for methylation variability. DeepCpG predicted more accurate end in comparison to other methods when evaluation using five differing kinds of methylation data. DNA methylation could be a most generally studied epigenetic marker (Angermueller et al .,2017).

Tensor Flow may be a deep learning framework developed by Google researchers. Tensor Flow could be a recently developed software that accelerates DNN design and training. it's implemented in several improvements like graphical visualization and time complication. Main improvement of TensorFlow that biology will still borrow from machine learning, or better still, machine learning will lead the way.

\section{APPLICATION OF DEEP LEARNING}

The deep learning contributions to the type of function areas in medical imaging. CNNs are put to task for classification, localization, detection, segmentation and registration in image analysis. Machine learning research draws a distinction between localization (draw a bounding box around one object within the image), and detection (draw bounding boxes around multiple objects, which can be from different classes). Segmentation draws outlines round the edges of target objects, and labels them (semantic segmentation). Registration refers to fitting one image (which is also 2 or 3 dimensional) onto another. Machine learning system will incorporate some or all of the tasks into a unified system. it'd be ideal to, in a very single workflow, detect a lung tumor on a CT chest scan, so localize and segment it far away from normal tissue, and to prognosticate various treatment options, like chemotherapy or surgery. From the clinician's perspective, classification ascertains if a disease state is present or not, i.e., is blood present on this MRI brain scan signifying a hemorrhagic stroke? Localization implies the identification of normal anatomy, as an example, where is that the kidney during this ultrasound image? this can be in contrast to detection, which suggests an abnormal, status, for instance, where are all the lung tumors during this CT scan of the lung? Segmenting the outline of a lung tumor helps the clinician determine its distance from major anatomical structures, and helps to answer a matter like, should this patient be operated on, and if so, what should be the extent of resection? 
Vol. 9, Issue 6, June 2020

A. Classification: Classification is usually also called Computer-Aided Diagnosis(CADx).

B. Localization: Localization of normal anatomy is a smaller amount likely to interest the practicing clinician although applications may arise in anatomy education. Alternatively, localization may find use in fully automated end-to-end applications, whereby the radiological image is autonomously analyzed and reported with none human intervention.

C. Detection: Detection, sometimes referred to as Computer-Aided Detection (CADe) may be a keen area of study as missing a lesion on a scan can have drastic consequences for both the patient and also the clinician.

D. Segmentation: CT and MRI mage segmentation research covers a range of organs like liver, prostate and knee cartilage, but an outsized amount of labor has focused on brain segmentation, including tumor segmentation. The latter is very important in surgical attending to determine the precise boundaries of the tumor so as to direct surgical resection. Sacrificing an excessive amount of of eloquent brain areas during surgery would cause neurological deficits like limb weakness, numbness and cognitive impairment. Traditionally, medical anatomical segmentation was done by hand, with a clinician drawing outlines slice by go a whole MRI or CT volume stack, therefore it's ideal to implement an answer that automates this laborious task.

E. Registration: Registration (i.e. spatial alignment) of medical images could be a common image analysis task within which a coordinate transform is calculated from one medical image to a different. Often this can be performed in an iterative framework where a selected form of (non-) parametric transformation is assumed and a pre-determined metric (e.g. L2-norm) is optimized. Although segmentation and lesion detection are more popular topics for deep learning, researchers have found that deep networks is beneficial in getting the simplest possible registration performance.

Broadly speaking, two strategies are prevalent in current literature: (1) using deep-learning networks to estimate a similarity measure for 2 images to drive an iterative optimization strategy, and (2) to directly predict transformation parameters using deep regression networks.

\begin{tabular}{|c|c|c|}
\hline \multirow[t]{2}{*}{ S.No } & \multicolumn{2}{|r|}{ Table 1:Deep learning techniques for brain image analysis. } \\
\hline & Method & Remarks \\
\hline 1 & ANN & $\begin{array}{l}\text { AD/MCI/HC classification; Multi-modal stacked deep polynomial networks with an SVM } \\
\text { classifier on top using MRI and PET (Shi et al., 2017). }\end{array}$ \\
\hline 2 & SAE & $\begin{array}{l}\text { MCI/HC classification of fMRI data; Stacked auto-encoders for feature extraction, HMM as a } \\
\text { generative model on top (Suk et al. ,2016) }\end{array}$ \\
\hline 3 & DBN & $\begin{array}{l}\text { Schizophrenia/NH classification; DBN pre-training followed by supervised fine-tuning (Pinaya } \\
\text { et al. 2016) }\end{array}$ \\
\hline 4 & CNN & $\begin{array}{l}\text { AD/MCI/HC classification; 3D CNN pre-trained with sparse auto-encoders (Payan and Montana } \\
\text { 2015). }\end{array}$ \\
\hline 5 & SAE & AD/MCI classification; Stacked auto encoders with supervised fine tuning (Suk and Shen, 2013) \\
\hline 6 & RBM & $\begin{array}{l}\text { AD/MCI/HC classification; Deep Boltzmann Machines on MRI and PET modalities (Suk et } \\
\text { al.,2014) }\end{array}$ \\
\hline 7 & DBN & $\begin{array}{l}\text { Deep belief networks evaluated on brain network estimation, Schizophrenia and Huntington's } \\
\text { disease classification (Plis et al., 2014) }\end{array}$ \\
\hline 8 & SAE & $\begin{array}{l}\text { MCI/HC classification of fMRI data; Stacked auto-encoders for feature extraction, HMM as a } \\
\text { generative model on top (Suk et al.,2016) }\end{array}$ \\
\hline
\end{tabular}

\begin{tabular}{|c|c|c|}
\hline \multirow[t]{2}{*}{ S.No } & \multicolumn{2}{|r|}{ Table 2: Deep learning techniques for retinal image analysis. All works use CNNs. } \\
\hline & Method & Remarks \\
\hline 1 & \multirow{6}{*}{ CNN } & $\begin{array}{l}\text { Glaucoma detection; end-to-end CNN, the input is a patch centered at the optic disk (Chen et } \\
\text { al.,2015) }\end{array}$ \\
\hline 2 & & $\begin{array}{l}\text { Diabetic retinopathy detection; end-to-end CNN, outperforms traditional method, evaluated on } \\
\text { a public dataset (Abramoff et al.,2016) }\end{array}$ \\
\hline 3 & & $\begin{array}{l}\text { Age-related macular degeneration detection; uses overfeat pretrained network for feature } \\
\text { extraction (Burlina et al.,2016) }\end{array}$ \\
\hline 4 & & $\begin{array}{l}\text { Hemorrhage detection; CNN dynamically trained using selective data sampling to perform hard } \\
\text { negative mining (van Grinsven et al. ,2016) }\end{array}$ \\
\hline 5 & & $\begin{array}{l}\text { Diabetic retinopathy detection; Inception network, performance comparable to a panel of seven } \\
\text { certified ophthalmologists (Gulshan et al.,2016). }\end{array}$ \\
\hline 6 & & Hard exudate detection; end-to-end CNN combined with the outputs of traditional classifiers \\
\hline
\end{tabular}


Vol. 9, Issue 6, June 2020

\begin{tabular}{|c|c|}
\hline & for detection of landmarks (Prentasic and Loncaric ,2016) \\
\hline 7 & $\begin{array}{l}\text { Retinopathy of prematurity detection; fine tuned ImageNet trained GoogLeNet, feature map } \\
\text { visualization to highlight disease (Worrall et al.,2016). }\end{array}$ \\
\hline 8 & $\begin{array}{l}\text { Glaucoma detection; end-to-end CNN, the input is a patch centered at the optic disk (Chen et } \\
\text { al.,2015). }\end{array}$ \\
\hline
\end{tabular}

\begin{tabular}{|c|c|c|}
\hline \multirow[t]{2}{*}{ S.No } & \multicolumn{2}{|r|}{ Table 3: Deep learning techniques for chest $x$-ray image analysis. } \\
\hline & Method & Remarks \\
\hline 1 & $\begin{array}{l}\text { Nodule } \\
\text { detection }\end{array}$ & $\begin{array}{l}\text { Classifies candidates from small patches with two layer CNN, each with } 125 \text { - } 5 \text { filters ( } \\
\text { Lo et al.,1995). }\end{array}$ \\
\hline 2 & Image retrieval & $\begin{array}{l}\text { Combines classical features with those from pre-trained CNN for image retrieval using } \\
\text { SVM (Anavi et al.,2015). }\end{array}$ \\
\hline 3 & $\begin{array}{l}\text { thology } \\
\text { tection }\end{array}$ & $\begin{array}{l}\text { Features from a pre-trained CNN and low level features are used to detect various } \\
\text { diseases (Bar et al., 2015). }\end{array}$ \\
\hline 4 & Image retrieval & $\begin{array}{l}\text { Continuation of Anavi et al. (2015), adding age and gender as features (Anavi et } \\
\text { al.,2016) }\end{array}$ \\
\hline 5 & $\begin{array}{l}\text { ggy } \\
\text { on }\end{array}$ & $\begin{array}{l}\text { Continuation of Bar et al. (2015), more experiments and adding feature selection (Bar et } \\
\text { al.,2016). }\end{array}$ \\
\hline 6 & $\begin{array}{l}\log y \\
\text { ion }\end{array}$ & $\begin{array}{l}\text { GoogLeNet CNN detects five common abnormalities, trained and validated on a large } \\
\text { data set (Cicero et al.,2016). }\end{array}$ \\
\hline 7 & $\begin{array}{l}\text { rculosis } \\
\text { tion }\end{array}$ & $\begin{array}{l}\text { Processes entire radiographs with a pre-trained fine-tuned network with } 6 \text { convolution } \\
\text { layers (Hwang et al. ,2016). }\end{array}$ \\
\hline 8 & $\begin{array}{l}\text { Tuberculosis } \\
\text { detection }\end{array}$ & $\begin{array}{l}\text { MIL framework produces heat map of suspicious regions via deconvolution (Kim and } \\
\text { Hwang ,2016). }\end{array}$ \\
\hline 9 & $\begin{array}{l}\text { Bone } \\
\text { suppression }\end{array}$ & $\begin{array}{l}\text { Cascade of CNNs at increasing resolution learns bone images from gradients of } \\
\text { radiographs(Yang et al., 2016). }\end{array}$ \\
\hline
\end{tabular}

\begin{tabular}{|c|c|c|}
\hline \multirow[t]{2}{*}{ S.No } & \multicolumn{2}{|r|}{ Table 4: Deep learning techniques for chest $\mathrm{CT}$ image analysis. } \\
\hline & Method & Remarks \\
\hline 1 & \multirow{5}{*}{$\mathrm{CT}$} & $\begin{array}{l}\text { Used a standard feature extractor and a pre-trained CNN to classify detected lesions as benign } \\
\text { peri-fissural nodules (Ciompi et al., 2015). }\end{array}$ \\
\hline 2 & & $\begin{array}{l}\text { Multi-stream CNN to classify nodules into subtypes: solid, part-solid, non-solid, calcified, } \\
\text { spiculated, perifissural (Ciompi et al.,2016). }\end{array}$ \\
\hline 3 & & $\begin{array}{l}\text { Detects nodules with end-to-end trained multi-stream CNN with } 9 \text { patches per candidate (Setio } \\
\text { et al., 2016). }\end{array}$ \\
\hline 4 & & $\begin{array}{l}\text { Combines features extracted from } 2 \text { orthogonal CT patches and a PET patch (Teramoto et } \\
\text { al.,2016) . }\end{array}$ \\
\hline 5 & & $\begin{array}{l}\text { 2D interstitial pattern classification with CNNs pre-trained with a variety of texture data sets } \\
\text { (Christodoulidis et al.,2017). }\end{array}$ \\
\hline
\end{tabular}

\begin{tabular}{|l|l|l|}
\hline \multirow{2}{*}{ S.No } & \multicolumn{2}{|c|}{ Table 5: Deep learning techniques for breast image analysis. } \\
\cline { 2 - 3 } & Method & Remarks \\
\hline 1 & MG CNN & Detection of cardiovascular disease based on vessel calcification (Wang et al.,2017). \\
\hline 2 & MG CNN & Semi-supervised CNN for classification of masses (Sun et al., 2016). \\
\hline 3 & MG CNN & $\begin{array}{l}\text { CNN for direct classification of future risk of developing cancer based on negative } \\
\text { mammograms (Qiu et al.,2016). }\end{array}$ \\
\hline 4 & MG CNN & Weakly supervised CNN for localization of masses (Hwang and Kim,2016) \\
\hline 5 & MG CNN & $\begin{array}{l}\text { Use a modified region proposal CNN (R-CNN) for the localization and classification of } \\
\text { masses (Akselrod-Ballin et al.,2016). }\end{array}$ \\
\hline 6 & MG, US ADN & Four layer ADN, an early form of CNN for mass classification (Jamieson et al.,2012). \\
\hline 7 & MG CNN & First application of a CNN to mammography (Sahiner et al., 1996). \\
\hline
\end{tabular}

\section{CONCLUSION}

Deep Learning may be a fast-moving and proliferating discipline. It's challenging to make a well-balanced and wellinformed summary view of the latest developments during this field. Thus, it's still harder to possess a vision of its future applications. Deep learning, also referred to as deep neural network learning may be a new and popular area of 
research that's yielding impressive results and growing fast. within the beginning, the models were simple and "brittle" that's, they failed to tolerate any deviations from the examples provided during training. Today's machine learning approaches are extremely robust to real-world conditions, and also the systems actually get pleasure from the forced dropout of some data within the learning process. because of the rapid pace of technologic advancements, tasks previously thought to be limited to humans are taken on by machine learning systems. Machine learning is already being applied within the practice of radiology, and these applications will probably grow at a rapid pace within the near future. the employment of machine learning in radiology has important implications for the practice of drugs, and it's important that we engage this area of research to make sure that the most effective care is afforded to patients. Understanding the properties of machine learning tools is critical to making sure that they're applied within the safest and handiest manner.

Many big research organization are acting on deep learning based solution that encourage to use deep learning to use deep learning on medical images. Looking to the brighter side of machine learning, we hope the earlier human are going to be replaced in most of the medical application especially diagnosis. However, we must always not consider it as only solution as there are several challenges that reduces its growth. One in the entire massive barrier is unavailability of annotated dataset. Thus, this question remains answerable, that whether we'll be able to get enough training data without effecting the performance of deep learning algorithms. Recent development on other application showed that bigger the info, better the result, however, how big data can be employed in healthcare.

\section{REFERENCES}

[1]. Abramoff, M. D., Lou, Y., Erginay, A., Clarida,W., Amelon, R., Folk, J. C., Niemeijer, M. Improved automated detection of diabetic Retinopathy on a publicly available dataset through integration of deep learning. Investigative Ophthalmology and Visual Science 57 (13), 5200-5206., 2016.

[2]. Akselrod-Ballin, A., Karlinsky, L., Alpert, S., Hasoul, S., Ben-Ari, R., Barkan, E. A region based convolutional network for tumor detection and classification in breast mammography. In: DLMIA. Vol. 10008 of Lecture Notes in Computer Science. pp. 197-205., 2016.

[3]. Anavi, Y., Kogan, I., Gelbart, E., Geva, O., Greenspan, H. A comparative study for chest radiograph image retrieval using binary texture and deep learning classification. In: Conference Proceedings of the IEEE Engineering in Medicine and Biology Society. pp. $2940-2943 ., 2015$.

[4]. Anavi, Y., Kogan, I., Gelbart, E., Geva, O., Greenspan, H. Visualizing and enhancing a deep learning framework using patients age and gender for chest X-ray image retrieval. In: Medical Imaging. Vol. 9785 of Proceedings of the SPIE. p. 978510., 2016

[5]. Angermueller, C., Lee, H. J., Reik, W., \& Stegle, O. DeepCpG: accurate prediction of single-cell DNA methylation states using deep learning. Genome biology, 18(1), 67, 2017.

[6]. Bar, Y., Diamant, I., Wolf, L., Greenspan, H. Deep learning with non-medical training used for chest pathology identification. In: Medical Imaging. Vol. 9414 of Proceedings of the SPIE. p. 94140V., 2015.

[7]. Bar, Y., Diamant, I., Wolf, L., Lieberman, S., Konen, E., Greenspan, H. Chest pathology identification using deep feature selection with nonmedical training. Computer Methods in Biomechanicsand Biomedical Engineering: Imaging \& Visualization, 1-5, ., 2016.

[8]. Burlina, P., Freund, D. E., Joshi, N., Wolfson, Y., Bressler, N. M. Detection of age-related macular degeneration via deep learning. In: IEEE International Symposium on Biomedical Imaging.pp. 184-188., 2016.

[9]. Chen, X., Xu, Y., Wong, D. W. K., Wong, T. Y., Liu, J. Glaucoma detection based on deep convolutional neural network. In:mConference Proceedings of the IEEE Engineering in Medicine and Biology Society. pp. 715-718., 2015.

[10]. Ching T, Himmelstein DS, Beaulieu-Jones BK, Kalinin AA, Do BT, Way GP, Ferrero E, Agapow P-M, Zietz M, Hoffman MM, et al: Opportunities and obstacles for deep learning in biology and medicine. Journal of The Royal Society Interface, 15:20170387, 2018.

[11]. Christodoulidis, S., Anthimopoulos, M., Ebner, L., Christe, A., Mougiakakou, S. Multi-source transfer learning with convolutional neural networks for lung pattern analysis. IEEE Journal of Biomedical and Health Informatics 21, 76-84., 2017.

[12]. Cicero, M., Bilbily, A., Colak, E., Dowdell, T., Gray, B., Perampaladas, K., Barfett, J. Training and validating a deep convolutional neural network for computer-aided detection and classification of abnormalities on frontal chest radiographs. Investigative Radiology, in press. ., 2016.

[13]. Ciompi, F., Chung, K., van Riel, S. J., Setio, A. A. A., Gerke, P. K., Jacobs, C., Scholten, E. T., Schaefer-Prokop, C. M.,Wille, M. M.W., Marchiano, A., Pastorino, U., Prokop, M., van Ginneken, B. Towards automatic pulmonary nodule management in lung cancer screening with deep learning. arXiv:1610.09157., 2016.

[14]. Ciompi, F., de Hoop, B., van Riel, S. J., Chung, K., Scholten, E. T., Oudkerk, M., de Jong, P. A., Prokop, M., van Ginneken, B. Automatic classification of pulmonary peri-fissural nodules in computed tomography using an ensemble of 2D views and a convolutional neural network out-of-the-box. Medical Image Analysis 26, 195-202., 2015.

[15]. Esteva A, Kuprel B, Novoa RA, Ko J, Swetter SM, Blau HM, Thrun S: Dermatologist-level classification of skin cancer with deep neural networks. Nature, 542:115-118, 2017.

[16]. Gulshan, V., Peng, L., Coram, M., Stumpe, M. C., Wu, D., Narayanaswamy, A., Venugopalan, S., Widner, K., Madams, T., Cuadros, J., Kim, R., Raman, R., Nelson, P. C., Mega, J. L., Webster, D. R., Dec.. Development and validation of a deep learning algorithm for detection of diabetic retinopathy in retinal fundus photographs. JAMA 316, 2402-24., 2016.

[17]. Hwang, S., Kim, H. Self-transfer learning for fully weakly supervised object localization. arXiv:1602.01625, 2016.

[18]. Hwang, S., Kim, H.-E., Jeong, J., Kim, H.-J. A novel approach for tuberculosis screening based on deep convolutional neural networks. In: Medical Imaging. Vol. 9785 of Proceedings of the SPIE. pp. $97852 \mathrm{~W}-1 ., 2016$.

[19]. Jamieson, A. R., Drukker, K., Giger, M. L. Breast image feature learning with adaptive deconvolutional networks. In: Medical Imaging. Vol. 8315 of Proceedings of the SPIE. p. $831506 ., 2012$.

[20]. Kevin Zhou S, Greenspan H \& Shen D. Deep Learning for Medical Image Analysis. Academic Press, 2017.

[21]. Kim, H., Hwang, S.,. Scale-invariant feature learning using deconvolutional neural networks for weakly-supervised semantic segmentation. arXiv:1602.04984, 2016.

[22]. Kotsiantis, S.B. "Supervised Machine Learning: A Review of Classification Techniques", Informatica 31,249-268, 2007.

[23]. LeCun Y, Bengio Y \& Hinton G .Deep learning. Nature 521, 436-444,2015.

[24]. Le Cun Y, Bottou L, Bengio Y, Haffner P. Gradient-based learning applied to document recognition. Proc IEEE; 86:2278-324, 1998.

[25]. Litjens G . A survey on deep learning in medical image analysis. Med. Image Anal 42, 60-88,2017. 
Vol. 9, Issue 6, June 2020

[26]. Lo, S.-C., Lou, S.-L., Lin, J.-S., Freedman, M. T., Chien, M. V., Mun, S. K. Artificial convolution neural network techniques and applications for lung nodule detection. IEEE Transactions on Medical Imaging 14, 711-718., 1995.

[27]. Mahmud, M., Kaiser, M. S., Hussain, A., \& Vassanelli, S. Applications of deep learning and reinforcement learning to biological data. IEEE transactions on neural networks and learning systems, 29(6), 2063-2079,2018.

[28]. Mamoshina P, Vieira A, Putin E, Zhavoronkov A: Applications of Deep Learning in Biomedicine. Molecular Pharma, 13:1445-1454, 2016.

[29]. Mnih V, Kavukcuoglu K, Silver D, Rusu A, Veness J, Bellemare M, Graves A, Riedmiller M, Fidjeland A, Ostrovski G, : Human-Level Control through Deep Reinforcement Learning. Nature, 518:529, 2015.

[30]. Payan, A., Montana, G., 2015. Predicting Alzheimer's disease: a neuroimaging study with 3D convolutional neural networks. arXiv: 1502.02506

[31]. Pinaya, W. H. L., Gadelha, A., Doyle, O. M., Noto, C., Zugman, A.,Cordeiro, Q., Jackowski, A. P., Suk, H.-I., Shen, D. Deep learning-based feature representation for AD/MCI classification. In: Medical Image Computing and Computer-Assisted Intervention. Vol. 8150 of Lecture Notes in Computer Science. pp. 583-590., 2016.

[32]. Plis, S. M., Hjelm, D. R., Salakhutdinov, R., Allen, E. A., Bockholt, H. J., Long, J. D., Johnson, H. J., Paulsen, J. S., Turner, J. A., Calhoun, V. D. Deep learning for neuroimaging: a validation study. Frontiers in Neuroscience., 2014.

[33]. Prentasic, P., Loncaric, S. Detection of exudates in fundus photographs using deep neural networks and anatomical landmark detection fusion. Computer Methods and Programs in Biomedicine 137, 281-292., 2016.

[34]. Qiu, Y., Wang, Y., Yan, S., Tan, M., Cheng, S., Liu, H., Zheng, B., An initial investigation on developing a new method to predict short-term breast cancer risk based on deep learning technology. In: Medical Imaging. Vol. 9785 of Proceedings of the SPIE. p. 978521.2016.

[35]. Sahiner, B., Chan, H.-P., Petrick, N., Wei, D., Helvie, M. A., Adler, D. D., Goodsitt, M. M. Classification of mass and normal breast tissue: a convolution neural network classifier with spatial domain and texture images. IEEE Transactions on Medical Imaging 15, 598-610., 1996.

[36]. Setio, A. A. A., Ciompi, F., Litjens, G., Gerke, P., Jacobs, C., van Riel, S., Wille, M. W., Naqibullah, M., Sanchez, C., van Ginneken, B. Pulmonary nodule detection in CT images: false positive reduction using multi-view convolutional networks. IEEE Transactions on Medical Imaging 35 (5), 1160-1169., 2016.

[37]. Shi, J., Zheng, X., Li, Y., Zhang, Q., Ying, S., Jan. Multimodal neuroimaging feature learning with multimodal stacked deep polynomial networks for diagnosis of Alzheimer's disease. IEEE Journal of Biomedical and Health Informatics, in press. 2017.

[38]. Shin H-C . Deep convolutional neural networks for computer-aided detection: CNN architectures, dataset characteristics and transfer learning. IEEE Trans. Med. Imag 35, 1285-1298,2016.

[39]. Suk, H.-I., Wee, C.-Y., Lee, S.-W., Shen, D. State-space model with deep learning for functional dynamics estimation in restingstate fMRI. NeuroImage 129, 292-307, 2016

[40]. Suk, H.-I., Lee, S.-W., Shen, D. Hierarchical feature representation and multimodal fusion with deep learning for AD/MCI diagnosis. NeuroImage 101, 569-582. 2014.

[41]. Sun, W., Tseng, T.-L. B., Zhang, J., Qian, W. Enhancing deep convolutional neural network scheme for breast cancer diagnosis with unlabeled data. Computerized Medical Imaging and Graphics. 2016.

[42]. Sutton, R. S. "Introduction: The Challenge of Reinforcement Learning", Machine Learning, 8, Page 225-227, Kluwer Academic Publishers, Boston, 1992

[43]. Teramoto, A., Fujita, H., Yamamuro, O., Tamaki, T. Automated detection of pulmonary nodules in PET/CT images: Ensemble false-positive reduction using a convolutional neural network technique. Medical Physics 43, 2821-2827., 2016.

[44]. Van Grinsven, M. J. J. P., van Ginneken, B., Hoyng, C. B., Theelen, T., S’anchez, C. I. Fast convolutional neural network training using selective data sampling: Application to hemorrhage detection in color fundus images. IEEE Transactions on Medical Imaging 35 (5), $1273-$ $1284,2016$.

[45]. Wang, J., Ding, H., Azamian, F., Zhou, B., Iribarren, C., Molloi, S., Baldi, P. Detecting cardiovascular disease from mammograms with deep learning. IEEE Transactions on Medical Imaging ., 2017.

[46]. Webb, S. Deep learning for biology. Nature. 554(7693):555-557, 2018

[47]. Worrall, D. E., Wilson, C. M., Brostow, G. J.,. Automated retinopathy of prematurity case detection with covolutional neural networks. In: DLMIA. Vol. 10008 of Lecture Notes in Computer Science. pp. 68-76.,2016.

[48]. Yang, W., Chen, Y., Liu, Y., Zhong, L., Qin, G., Lu, Z., Feng, Q., Chen, W. Cascade of multi-scale convolutional neural networks for bone suppression of chest radiographs in gradient domain. Medical Image Analysis 35, 421-433., 2016.

[49]. Zhang S, Zhou J, Hu H, Gong H, Chen L, Cheng C, Zeng J: A Deep Learning Framework for Modeling Structural Features of RNA-Binding Protein Targets. Nucleic Acids Res, $44: \mathrm{e} 32,2016$. 